\title{
Preparation and Characterization of Nano Titanium Dioxide Photocatalysts via Sol Gel Method over Narrow Ranges of Varying Parameters
}

\author{
DOAA M. EL-MEKKAWI ${ }^{1 *}$, AMMAR A. LABIB ${ }^{2}$, HANAN A. MOUSA ${ }^{2}$, \\ HODA R. GALAL ${ }^{2}$ and WALIED A. A. MOHAMED ${ }^{2}$ \\ ${ }^{1}$ Physical Chemistry Department, National Research Centre, \\ NRC, 33 EL Bohouth st.- Dokki-Giza- Egypt, P. O. 12622. \\ ${ }^{2}$ Inorganic Chemistry Department, National Research Centre, \\ 33 Elbohouth st.- Dokki-Giza- Egypt. P. O. 12622. \\ *Corresponding author E-mail: doaa_egypt@yahoo.com \\ http://dx.doi.org/10.13005/ojc/330105
}

(Received: January 01, 2017; Accepted: February 12, 2017)

\begin{abstract}
The present study addresses a tailored preparation method of different structures of $\mathrm{TiO}_{2}$ nanoparticles over narrow ranges of varying parameters. In this work, titanium-n-butoxide was used to prepare $\mathrm{TiO}_{2}$ nanoparticles via sol gel method. The influence of the experimental preparation conditions: $\mathrm{pH}$, drying and calcination temperatures were studied at ranges $5-9,70-110^{\circ} \mathrm{C}$, and $450-650^{\circ} \mathrm{C}$, respectively. Slight changes through these mentioned ranges lead to drastically change in the phase transformation, degrees of crystallinity and crystal systems of the prepared $\mathrm{TiO}_{2}$. Pure anatase and rutile forms as well as different ratios of mixed phases were obtained. X-ray diffraction and transmitting electron microscope TEM techniques were used for the characterization of the prepared samples. The photocatalytic performance of $\mathrm{TiO}_{2}$ samples were evaluated according to their abilities toward the generation of the highly active hydroxyl radicals. Results indicate that the photocatalytic activities of $\mathrm{TiO}_{2}$ samples are dependent on their preparation experimental conditions. The maximal photocatalytic activities were noticed at high $\mathrm{pH}$ and drying temperature values.
\end{abstract}

Keywords : $\mathrm{TiO}_{2}$ preparation; sol gel; $\mathrm{TiO}_{2}$ characterization; photocatalytic activities; fluorescent probe method.

\section{INTRODUCTION}

Titanium dioxide is exceedingly considered as one of the most promising semiconductors. Its application areas cover a wide variety of different disciplines including both industrial and academic sectors $^{1-12}$.

Preparation of the photocatalytically active crystalline phases; anatase and rutile of $\mathrm{TiO}_{2}$ has been previously interpreted through different 
techniques. These techniques include the sol-gel ${ }^{13-}$ 14, hydrothermal method ${ }^{15}$, thermal hydrolysis ${ }^{16}$, solvothermal ${ }^{17}$, direct oxidation ${ }^{18}$, electrodeposition ${ }^{19}$, micelle and inverse micelle usage ${ }^{20}$, emulsion or hydrolysis precipitation ${ }^{21}$, chemical/physical vapor deposition ${ }^{22}$, microemulsion processes ${ }^{23,24}$, microwave approaches and ultrasonic ${ }^{25,26}$. These numerous studies addressed huge number of varieties including materials, experimental tools, instrumentations and experimental conditions. Accordingly, researchers have to go through various preparation methods to get $\mathrm{TiO}_{2}$ samples of certain desired characteristics. This may consequently add some difficulties especially in case of the lack of resources.

Among the numerous preparation techniques, sol gel is considered as one of the simple methods for synthesizing nanoparticles at ambient conditions. Besides, it does not require complicated set-up and the experimental conditions can easily be controlled ${ }^{27}$.

In our previous work, evaluation of the photocatalytic activities of $\mathrm{TiO}_{2}$ in the efficient removal of different organic pollutants was conducted under identical conditions ${ }^{28,29}$. It was found that the performance of $\mathrm{TiO}_{2}$ was significantly affected by their capabilities of the surface hydroxyl radicals' generation. Accordingly, $\mathrm{TiO}_{2}$ were divided into two categories: HRT and LRT; representing the photocatalysts that possess high and low rates of the hydroxyl radical formation, respectively. It was found that efficient removal of pollutants was achieved by using HRT photocatalysts whenever there is no surface interaction hindrance. However, some of $\mathrm{TiO}_{2}$ samples that belong to LRT group showed remarkable selectivity to certain pollutants. The amount of surface hydroxyl radicals' generated by $\mathrm{TiO}_{2}$ particles is consistently influenced by their physicochemical characteristics. These characteristics include crystal structure, morphology, crystallite size, phase composition, particle aggregation, calcination degrees and surface hydroxyl group content ${ }^{30-33}$.

In the present work, preparation of different structures of $\mathrm{TiO}_{2}$ nanoparticles over narrow ranges of varying parameters is investigated. In this study, $\mathrm{TiO}_{2}$ was prepared from titanium tetran-butoxide via sol-gel method. Preparation of $\mathrm{TiO}_{2}$ via sol-gel method occurs through hydrolysis of titanium alkoxide followed by polycondensation process $^{34}$. Previously, it was found that the anatase phase began to appear upon the calcination of the hydrolyzed species of titanium alkoxides at $450^{\circ} \mathrm{C}$ for 3 hours ${ }^{35}$. However, slight change in parameters such as $\mathrm{pH}$, drying temperature, ionic species, calcination temperature and time significantly affect the $\mathrm{TiO}_{2}$ characteristics. Therefore, different crystallinities and structures of $\mathrm{TiO}_{2}$ can be achieved by controlling such parameters. The main target of this study is to prepare different possible ratios of rutile and anatase as photocatalytically active phases with different crystal properties. The photocatalytic activities of the prepared $\mathrm{TiO}_{2}$ nanoparticles were then evaluated with respect to their abilities to generate surface active hydroxyl radicals.

\section{MATERIAL AND METHODS}

All the chemicals were of analytical grade and were used without further purification. Titanium tetra- n-butoxide was obtained from Aldrich. Water used in this investigation was de-ionized water. Acetic acid, ammonia and propanol were purchased from Merck company.

\section{Preparation of nano titanium dioxide}

Titanium dioxide nanoparticles were synthesized by sol- gel technique using titanium alkoxide as precursor ${ }^{35-39} .20 \mathrm{ml}$ of titanium tetrabutoxide was mixed with $40 \mathrm{ml}$ of 2-propanol in dry atmosphere. The mixture was added dropwise into another cold mixture of $20 \mathrm{ml}$ deionized water and $20 \mathrm{ml}$ of 2-propanol. The $\mathrm{pH}$ of the reaction media was adjusted by adding acetic acid or ammonia into the solutions. The addition time varied from $10 \mathrm{sec}$ to 45 min with stirring at $400 \mathrm{RPM}$. The $\mathrm{pH}$ values were adjusted to 2, 7 and 9 and the corresponding samples are $\mathrm{Ac}, \mathrm{Nu}$, and $\mathrm{Ak}$, respectively. The gel was dried in an air circulating oven at different temperatures overnight. (Ac1, Nu1, Ak1), (Ac2, Nu2, Ak3) and (Ac3, Nu3, Ak3) represent the dehydrated groups at $70^{\circ} \mathrm{C}, 90^{\circ} \mathrm{C}$ and $110^{\circ} \mathrm{C}$, respectively. Finally, the dry gels were calcined in preheated muffle furnace at 450,550 and $650^{\circ} \mathrm{C}$ for 3 hours.

\section{Characterization of $\mathrm{TiO}_{2}$ samples}

The crystallinity of titanium dioxide powder was determined by $\mathrm{X}$-ray diffraction using Ni-filled $\mathrm{Cu}$ 
$K \alpha$ radiation using Bruker $D 8$ Advanced Instrument (Germany D8 ADVANCE Cu target $1.54 \AA$, 40 kV, $40 \mathrm{~mA}$ ). The reference data for the interpretation of the X-ray diffraction patterns were obtained from the International Centre for Diffraction Data - Joint Committee on Powder Diffraction Standards ICDDJCPDS card files. The crystallite size (D) determined by the Scherrer's formula $(D=K \lambda /(b \cos \theta)) \cdot \lambda$ is the wavelength of $X$ - ray radiation (CuK $\alpha=0.15406 \mathrm{~nm})$, $\mathrm{K}$ is a constant taken as $0.89, \mathrm{~b}$ is the line width at half maximum height (FWHM) of the peak, and $\theta$ is the diffracting angle ${ }^{40}$.

The samples examined in the transmission electron microscope (TEM) were prepared by dispersing these samples in distilled water followed by ultrasonic vibration for half an hour. A drop of the dispersed sample was placed onto a copper grid coated with a layer of amorphous carbon. A JEOL transmission electron microscope, Philips $\mathrm{CM}-120$ was used to study the shape and particle size of $\mathrm{TiO}_{2}$ samples.

\section{Photocatalytic activity evaluation of $\mathrm{TiO}_{2}$ samples}

The photocatalytic activities of $\mathrm{TiO}_{2}$ samples with respect to their abilities to generate surface active hydroxyl radicals were evaluated via the fluorescent probe method ${ }^{41}$. Illumination of the $\mathrm{TiO}_{2}$ nanoparticles were carried out using the UVA light bulbs (15 W). The light intensity was measured by YK-35UV radiometer, Taiwan, and it was 2.60 $\mathrm{mW} / \mathrm{cm}^{2}$. The UV photoreactor lamps emitted the photons in the range from 320 to $410 \mathrm{~nm}$. The photoluminescence spectra were measured using JASCO spectrophotometer ( v-570, Rel-00, Japan). The apparent rate constant $\left(\mathrm{k}_{\mathrm{f}}\right)$ was calculated using the slope of the fluorescence intensity-illumination time curves.

\section{RESULTS AND DISCUSSION}

The nano titanium dioxide samples were prepared from titanium tetra- $n$-butoxide in three different pH's, 2, 7 and 9. Acetic acid and ammonium solutions were used as hydrolyzing agents in $\mathrm{pH}$ 's, 2 and 9, respectively. The samples were left to stir at $400 \mathrm{rpm}$. The washed precipitates were dried in oven at different temperature 70,90 and $110^{\circ} \mathrm{C}$ overnight. The samples were then calcined at three temperatures 450,550 and $650{ }^{\circ} \mathrm{C}$ for three hours. The calcined samples were directly investigated by using XRD, TEM, and fluorescence spectroscopy.

\section{XRD measurements}

The XRD difractograms of the titanium dioxide indicate the formation of anatase and rutile phases with different degrees of crystallinity. As shown in figures 1,2 and 3 , the anatase phase consists of five characteristic peaks at $2 \theta=25.28$, $36.00,36.96,37.60,48.00,53.92,55.04,62.40$ and 62.72 degrees. Meanwhile, in case of rutile form, the main characteristic peaks appear at $2 \theta=27.36,36.1639 .04,41.28,54.40,56.64$, 62.72 and 64.16 degrees. The experimental XRD data agree with the JCPDS card No. 84-1286 (anatase $\mathrm{TiO}_{2}$ ) and 87-0920 (rutile $\mathrm{TiO}_{2}$ ) [42, 43]. The characteristic peaks were analyzed for all $\mathrm{TiO}_{2}$ samples. Calculated degrees of crystallinity and crystal sizes as well as the type of the formed phases of each prepared sample are summarized in table 1.

Fig 1 illustrates the XRD diffractograms of the prepared $\mathrm{TiO}_{2}$ samples calcined at $450^{\circ} \mathrm{C}$. As indicated in table 1 and fig 1 , only anatase phases were obtained at all of the given experimental conditions. At this low calcination temperature, the formation of anatase as a kinetically stable phase is expected rather than rutile phase ${ }^{44}$. The calculated percentages of degrees of crystallinity were low and having the same values $(=50 \%)$, see table 1 . No significant variations were observed in the calculated crystal sizes in both acidic and neutral media at drying temperatures from 70 to $110^{\circ} \mathrm{C}$. However, slight increase in the crystal sizes was obtained in the alkaline media which is more significant at lower drying temperatures. This is probably due to the presence of the large amount of hydroxyl ions. The high concentrations of the hydroxyl ions result in rapid precipitation of the hydrolyzed titania species leading to their agglomeration. At high drying temperature, crystallization was probably initiated forming agglomerates consisting of several crystals upon drying. Contrary, samples dried at low temperature remained amorphous agglomerates. These amorphous agglomerates tend to form relatively large single crystals upon the calcination at $450{ }^{\circ} \mathrm{C}^{45}$. 
Generally, the formation mechanism of $\mathrm{TiO}_{2}$ from titanium alkoxides involves two main steps. The first step is the hydrolysis of the 4-coordinated $\left[\mathrm{Ti}(\mathrm{OR})_{4}\right]$ in water. In this step, titanium ion accepts oxygen lone pairs through the vacant d-orbitals yielding $\left[\mathrm{Ti}\left(\mathrm{H}_{2} \mathrm{O}\right)_{6}\right]^{4+}$ and consequently increases its coordination. The formed hydrolyzed species then undergo partial deprotonations and for simplicity is written as $\left(\mathrm{TiO}_{6}{ }^{2-}\right)$ octahedra. The second step is condensation. In this step, both anatase and rutile grow from $\mathrm{TiO}_{6}{ }^{2-}$ octahedra. Rutile consists of linear chains, formed by sharing two opposite edges of adjacent $\mathrm{TiO}_{6}$ octahedra, which are linked to other chains through shared corners. Anatase shares two sets of two adjacent edges to form zigzag chains linked to one another ${ }^{34}$. Therefore, the experimental conditions at which the arrangements of $\mathrm{TiO}_{6}$ octahedra occur should be precisely controlled so the desired $\mathrm{TiO}_{2}$ phase(s) could be obtained.

As shown in table 1, growth in the crystallite sizes was observed for $\mathrm{TiO}_{2}$ calcined at $550^{\circ} \mathrm{C}$. The calculated percentages of degrees of crystallinity remain low in basic and also in acidic medium at low drying temperatures. However, they increased to $100 \%$ for all $\mathrm{TiO}_{2}$ samples prepared at neutral and also for samples prepared in acidic medium at high drying temperature; $110^{\circ} \mathrm{C}$. Characteristic peaks of rutile phase began to appear in some samples (see fig 2 and table 1). Only anatase phases were obtained for those samples prepared at low degrees of crystallinity (i.e. Ac1, Ac2, Ak1, Ak2 and Ak3). Meanwhile, rutile phases appear in the samples with $100 \%$ degrees of crystallinity (i.e. Ac3, Nu1, Nu2 and Nu3). The percentage of rutile is 8.2 in sample prepared in acidic medium at high drying temperature (Ac3). The percentages of rutile are 33.2, 8 and 5.5 in samples prepared in neutral media dried at 70,90 and $110{ }^{\circ} \mathrm{C}$, respectively. The existence of anions in the reaction media plays an important role in the mobility and rearrangement of $\mathrm{TiO}_{6}$ species ${ }^{44,46}$. The hydroxyl and acetate anions in the basic and acidic solutions, respectively, have strong affinities toward titanium. The adsorption of these anions decreases the mobility of titanium oxyhydroxide moieties and also retards their arrangement into the rutile shape. These consequently result in low crystallinity of the prepared samples and hinder the formation of the thermodynamically stable rutile phase. These were obviously noticed in sampled prepared in basic and also in acidic medium at low drying temperatures. Contrary, the high drying temperature at $\mathrm{pH} 2$, results in vaporization of acetic acid. This in turn facilitates the mobility and arrangement of $\mathrm{TiO}_{6}$ moieties and consequently enhances both crystallinity and rutile formation. On the other hand, at $\mathrm{pH} 7$, the

Table 1: Crystal characterization of $\mathrm{TiO}_{2}$ samples estimated from the XRD analyses

\begin{tabular}{|c|c|c|c|c|c|c|c|c|c|c|}
\hline \multirow[t]{2}{*}{ Temp. } & & \multicolumn{3}{|c|}{ Acidic (Ac) pH2 } & \multicolumn{3}{|c|}{ Neutral $(\mathrm{Nu}) \mathrm{pH} 7$} & \multicolumn{3}{|c|}{ Alkaline (Ak) pH9 } \\
\hline & & Ac1 & Ac2 & Ac3 & Nu1 & Nu2 & Nu3 & Ak1 & Ak2 & Ak3 \\
\hline \multirow[t]{4}{*}{$450^{\circ} \mathrm{C}$} & Degree of crystallinity $\%$ & 50 & 50 & 50 & 50 & 50 & 51.8 & 50 & 50 & 50 \\
\hline & Anatase, \% & 100 & 100 & 100 & 100 & 100 & 100 & 100 & 100 & 100 \\
\hline & Rutile, \% & - & - & - & - & - & - & - & - & - \\
\hline & Anatase crystal size,nm & 18.25 & 15.40 & 15.60 & 13.00 & 13.50 & 14.80 & 57.56 & 29.48 & 18.08 \\
\hline \multirow[t]{5}{*}{$550^{\circ} \mathrm{C}$} & Degree of crystallinity \% & 50 & 50 & 100 & 100 & 100 & 100 & 50 & 50 & 50 \\
\hline & Anatase, \% & 100 & 100 & 91.8 & 66.8 & 91.9 & 94.5 & 100 & 100 & 100 \\
\hline & Rutile, \% & - & - & 8.2 & 33.2 & 8.1 & 5.5 & - & - & - \\
\hline & Anatase crystal size,nm & 33.18 & 37.02 & 41.70 & 58.53 & 44.00 & 40.74 & 47.60 & 44.00 & 26.80 \\
\hline & Rutile crystal size,nm & - & - & 42.16 & 78.90 & 74.32 & 66.40 & - & - & - \\
\hline \multirow[t]{5}{*}{$650^{\circ} \mathrm{C}$} & Degree of crystallinity $\%$ & 100 & 100 & 100 & 100 & 100 & 100 & 100 & 100 & 100 \\
\hline & Anatase, \% & 100 & 68 & 37 & - & 26.9 & 49 & 61.9 & 48.8 & 15 \\
\hline & Rutile, \% & - & 32 & 63 & 100 & 73.1 & 51 & 38.1 & 51.2 & 85 \\
\hline & Anatase crystal size,nm & 68.35 & 87.40 & 89.60 & - & 63.48 & 71.87 & 65.90 & 70.52 & 80.53 \\
\hline & Rutile crystal size,nm & - & 114.20 & 98.80 & 90.97 & 71.88 & 67.75 & 134.46 & 115.28 & 108.14 \\
\hline
\end{tabular}

Dried at: $1=70^{\circ} \mathrm{C} ; 2=90^{\circ} \mathrm{C} ; 3=110^{\circ} \mathrm{C}$; 
absence of anions results in facile arrangement of $\mathrm{TiO}_{6}$ moieties to form the rutile shape. The water to titanium oxyhydroxide ratios also play a significant role in the shape samples prepared in neutral media.
As water ratios decrease upon raising the drying temperatures, the mobility of titanium oxyhydroxide precursors decreases. The low mobility results in low interaction between the reaction precursors and

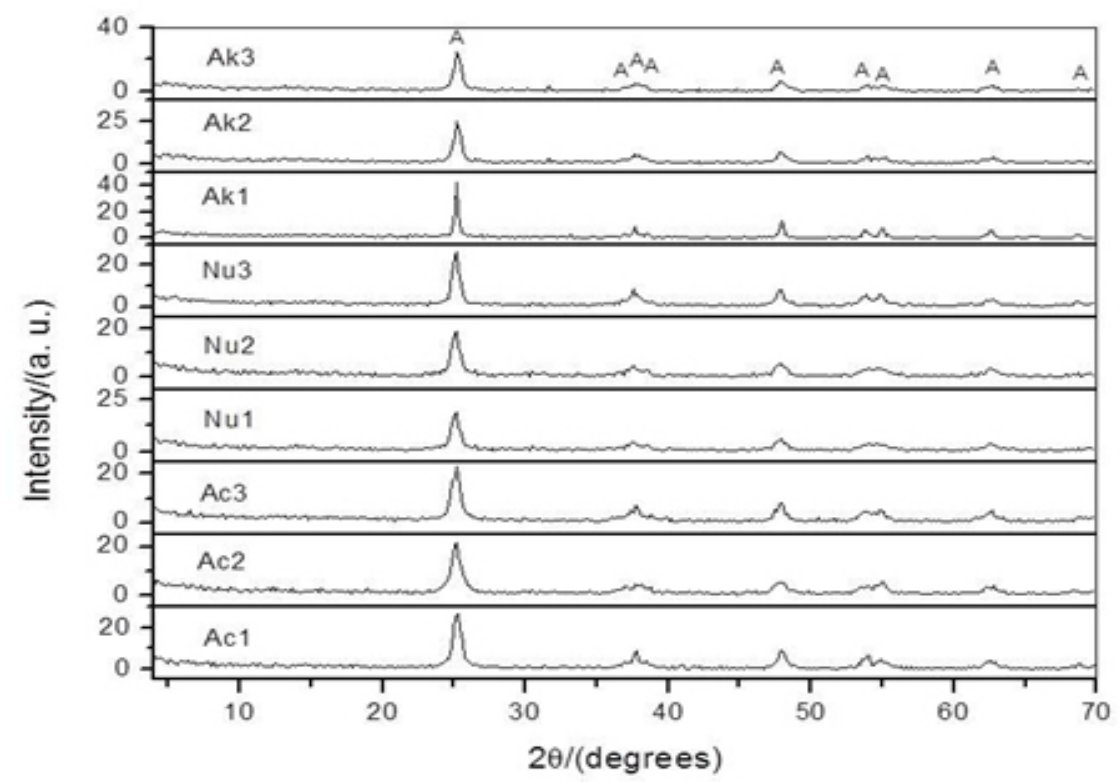

Fig .1: X-ray difractograms of $\mathrm{TiO}_{2}$ samples calcined at $450{ }^{\circ} \mathrm{C} . \mathrm{A}=$ anatase

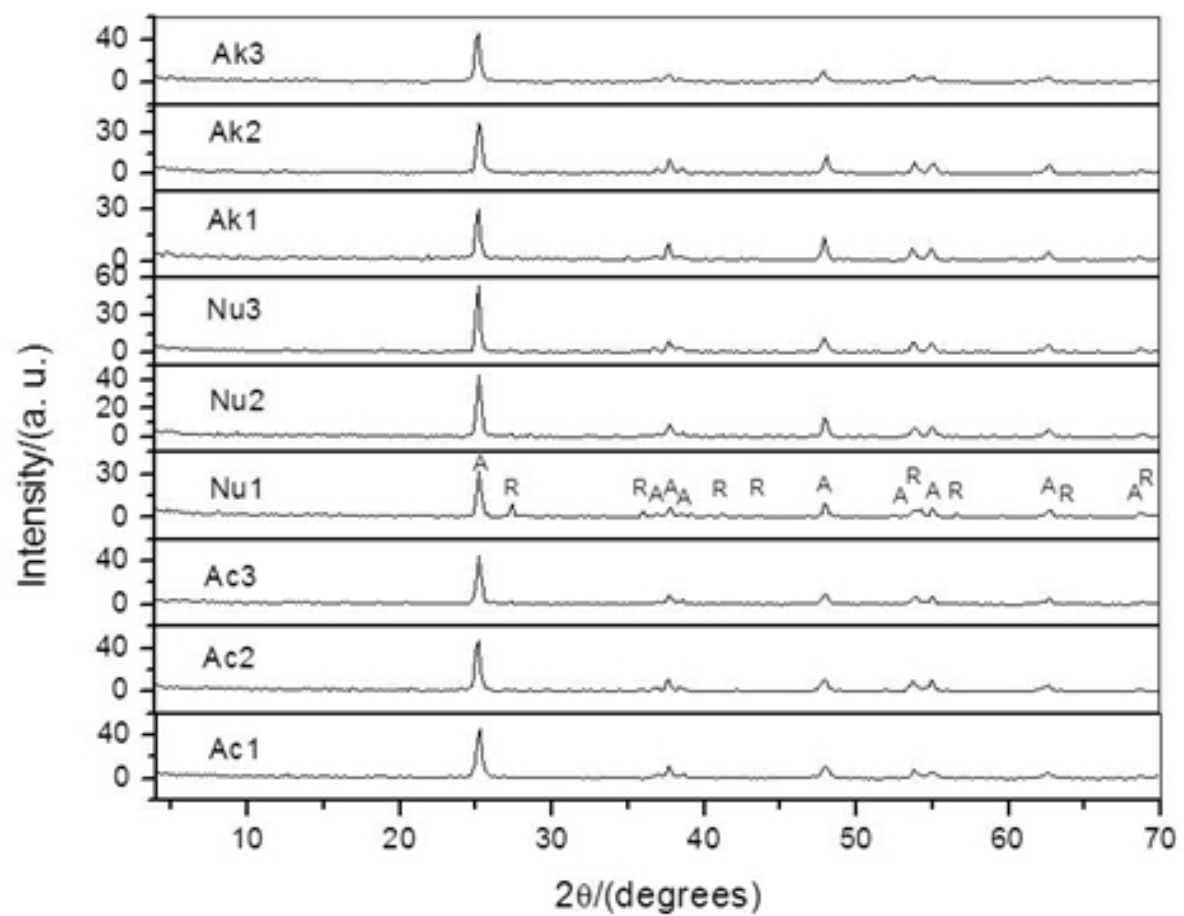

Fig. 2: X-ray difractograms of $\mathrm{TiO}_{2}$ samples calcined at $550^{\circ} \mathrm{C} . \mathrm{A}=$ anatase and $\mathrm{R}=$ rutile 
consequently results in the formation of $\mathrm{TiO}_{2}$ with low rutile ratios. Here again at high drying temperatures, crystallization was assumed to be initiated forming several crystals upon drying causing the overall decrease in the crystal sizes.

Further increase in the crystallite sizes was observed in samples calcined at $650^{\circ} \mathrm{C}$. The degrees of crystallinity of the prepared samples also increased to $100 \%$. Rutile phase appeared in most samples. Pure rutile phase was obtained at low drying temperature in neutral medium. However the rutile ratios decrease upon increasing the drying temperatures. The absence of anions and the water to titanium oxyhydroxide ratios significantly affect the rutile ratios as discussed above. However, the rutile ratios increase upon increasing the drying temperatures on both acidic and basic media. This is due to the vaporization of undesired anions as mentioned before. Contrary to that obtained at calcination temperatures, 450 and $550^{\circ} \mathrm{C}$, the crystal sizes of anatase phases increase upon increasing the drying temperatures. The small anatase crystals formed at high drying temperatures collapsed upon calcination at $650^{\circ} \mathrm{C}$ forming $\mathrm{TiO}_{2}$ crystals with larger dimensions. Findings are different with respect to the crystal sizes of rutile phases. The crystal sizes decrease upon increasing the drying temperatures.
We suppose that the small crystals initiated at high drying temperatures undergo rearrangements to form rutile phase rather than growing into larger crystals.

\section{Transmission electron microscopy (TEM) analyses}

The morphology and the particle size distribution of prepared titanium dioxide samples were characterized by TEM, fig. 4. TEM measurements of all of the prepared samples were carried out (not all are given). Overall, the measured TEM micrographs are in accordance with data obtained from the XRD analyses. TEM images represent different shapes, sizes and crystallinities of the prepared $\mathrm{TiO}_{2}$ samples according to their experimental conditions. Four of TEM images have been selected and shown in fig. 4. The four images represent anatase, rutile and mixed phases of $\mathrm{TiO}_{2}$ with different particle sizes. The anatase face appeared in its characteristic (001) cube face. The rutile shape appeared in its characteristic (011) hexagonal face [47]. For example, fig $4 \mathrm{a}$ represents the TEM image of Nu1 prepared at $450^{\circ} \mathrm{C}$. In fig. $4 \mathrm{a}$, anatase phase appears with particle sizes ranging from 5 to $25 \mathrm{~nm}$. As shown in fig $4 \mathrm{a}$, the anatase particles are dispersed over amorphous matrix. This amorphous matrix may be the unreacted titanium butoxide or amorphous

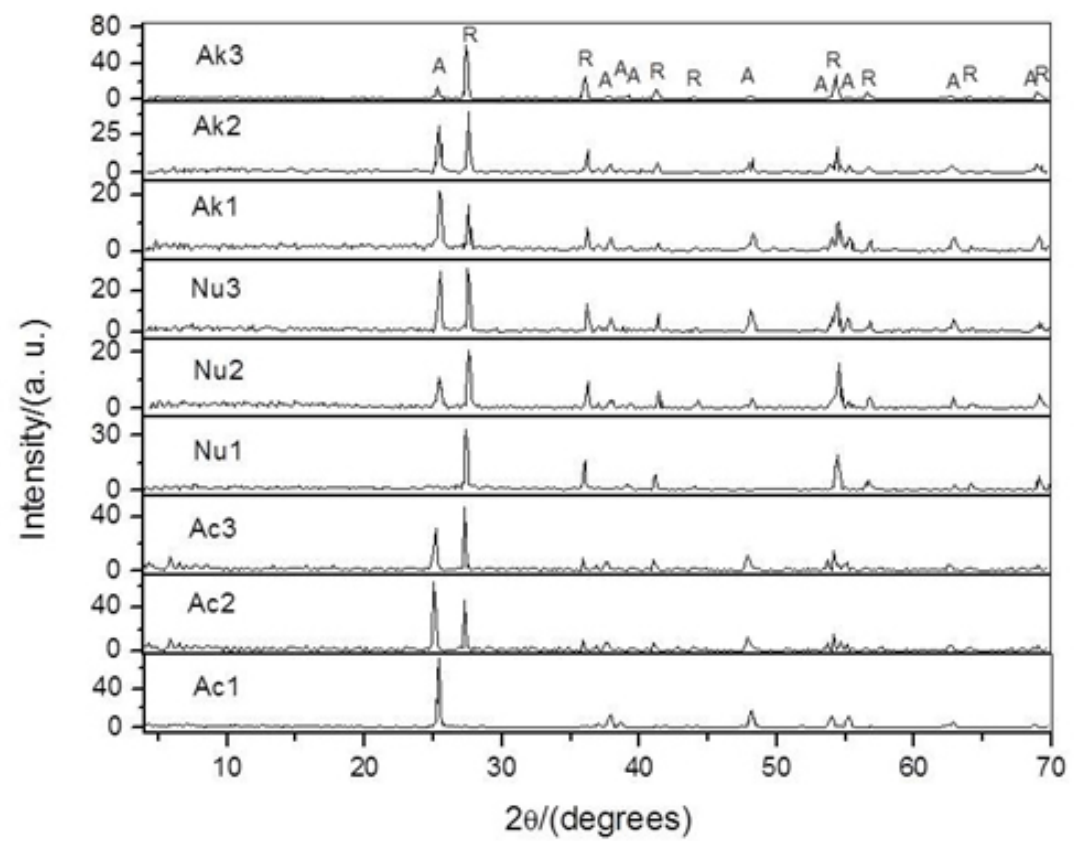

Fig. 3: X-ray difractograms of $\mathrm{TiO}_{2}$ samples calcined at $650^{\circ} \mathrm{C} . \mathrm{A}=$ anatase and $\mathrm{R}=$ rutile 
titanium oxyhydroxide species, etc. These findings are in consistent with the XRD data of Nu1 where the detected phase was anatase of $50 \%$ degree of crystallinity. Fig $4 b$ represents the TEM image of Nu3 prepared at $550{ }^{\circ} \mathrm{C}$. In fig $4 \mathrm{~b}$, well organized shapes of $\mathrm{TiO}_{2}$ mixed phases appear with the predominance of the anatase phase (cube shapes). The measured particle sizes obtained from TEM are ranging from 35 to $60 \mathrm{~nm}$ for anatase and from 55 to $70 \mathrm{~nm}$ for rutile. XRD data previously revealed that Nu3 prepared at $550^{\circ} \mathrm{C}$ consists of anatase and rutile mixture of percentages 94.5 and $5.5 \%$, respectively. Fig $4 \mathrm{c}$ represents the TEM image of Ac2 at $650^{\circ} \mathrm{C}$. TEM indicates that in Ac2 is a mixture of rutile and anatase phases. The particle sizes are ranging from 45 to $80 \mathrm{~nm}$ for anatase and from 40 to $120 \mathrm{~nm}$ for rutile. This is also in accordance with data obtained from XRD. XRD indicated the existence of anatase and rutile phases with percentages 68 and $32 \%$, respectively. Finally, TEM analyses also confirmed the XRD data of Nu1 prepared at $650{ }^{\circ} \mathrm{C}$ (fig. $4 d$ ). Only pure well-crystalline rutile phase appears in Nu1 (i.e. hexagonal shape). The measured particle sizes derived from TEM measurements ranges from 45 to $85 \mathrm{~nm}$.

\section{Photocatalytic activity evaluation of $\mathrm{TiO}_{2}$ samples}

Fluorescent probe method has been used for initial evaluation of the photocatalytic activity of the $\mathrm{TiO}_{2}$ samples calcined at $550{ }^{\circ} \mathrm{C}$. $\mathrm{TiO}_{2}$ samples calcined at $550{ }^{\circ} \mathrm{C}$ have been selected as they show different shapes and crystallinities. The $\left({ }^{\circ} \mathrm{OH}\right)$ produced at $\mathrm{TiO}_{2}$ surfaces upon UV light irradiation results in the conversion of non-fluorescent coumarin to 7-hydroxycoumarin fluorescent species ${ }^{41,48,49}$. The rates by which the coumarin molecules are converted to hydroxycoumarin $\left(k_{f}\right)$ measure the photocatalytic activities of $\mathrm{TiO}_{2}$ samples. The high rates of hydroxycoumarin formation indicate the high
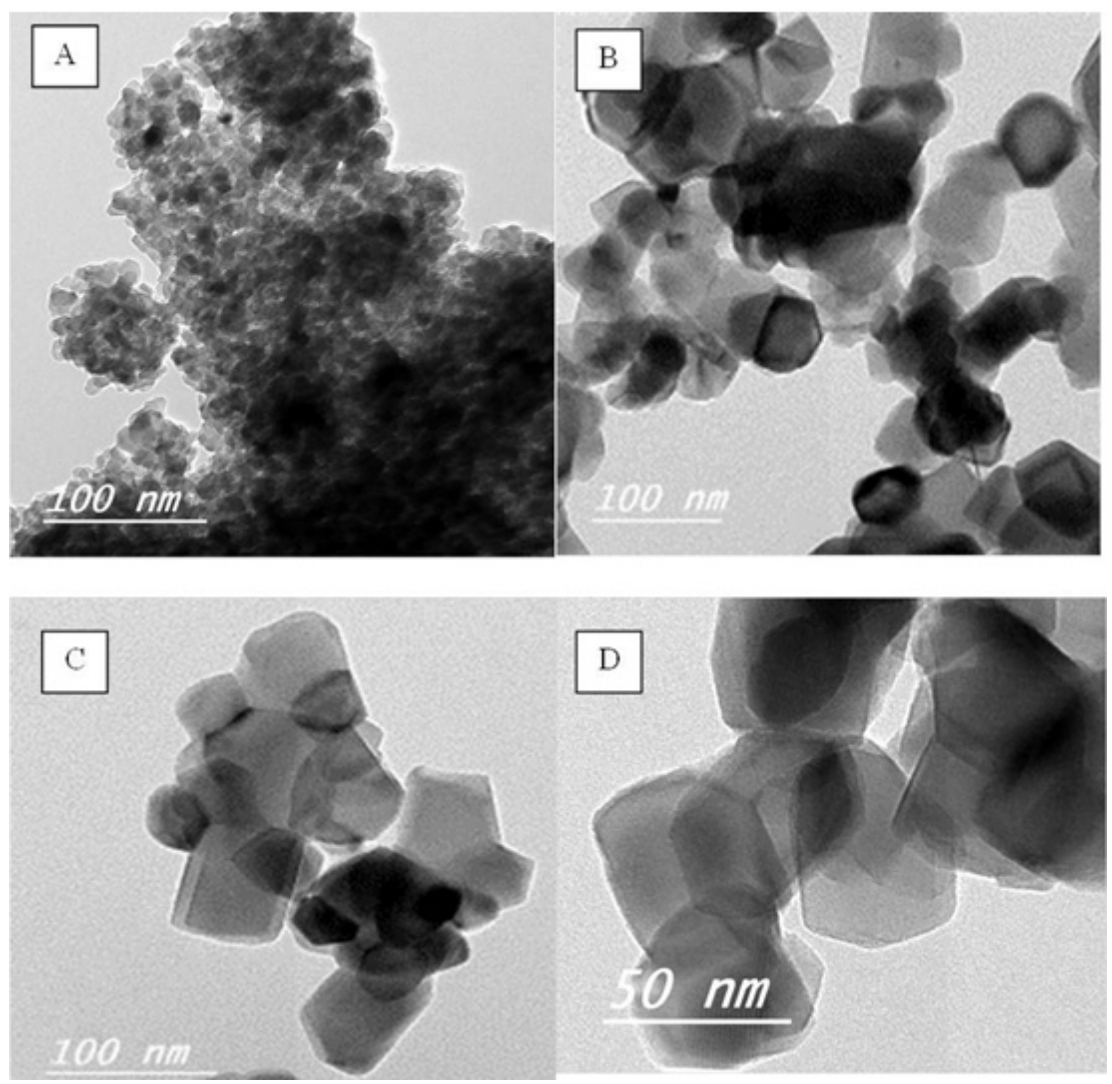

Fig. 4: TEM micrographs of $\mathrm{TiO}_{2}$ nanoparticles prepared at different experimental conditions. A: Nu1 at $450^{\circ} \mathrm{C}, \mathrm{B}$ : Nu3 at $550^{\circ} \mathrm{C}, \mathrm{C}$ : Ac2 at $650^{\circ} \mathrm{C}$, and D: Nu1 at $650^{\circ} \mathrm{C}$ 
abilities of $\mathrm{TiO}_{2}$ to produce surface hydroxyl radicals and consequently refer to their high photocatalytic performances.

Figure 5 a shows the change in the fluorescence spectrum of coumarin by UV light at different illumination time intervals. As shown in Fig $5 a$, peaks with maximum fluorescence intensities at $452 \mathrm{~nm}$ appeared upon UV light illumination. These new peaks refer to the formation of 7-hydroxycoumarin fluorescent species. The fluorescence intensities at $452 \mathrm{~nm}$ were plotted

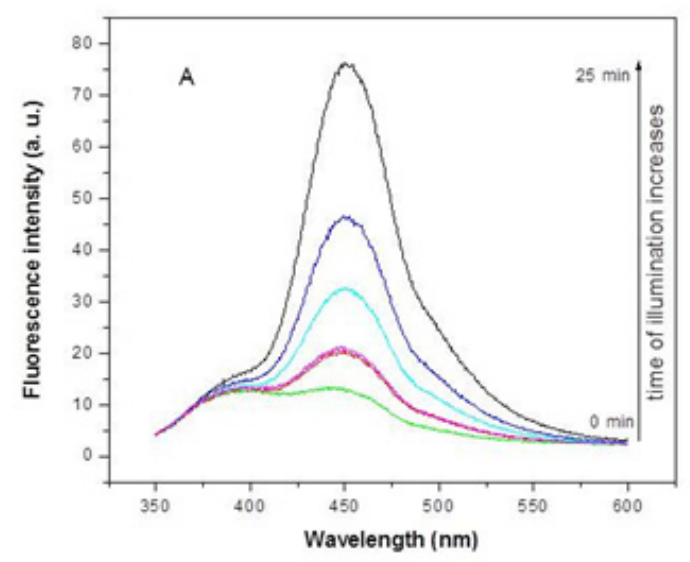

against the time of illumination for the different $\mathrm{TiO}_{2}$ samples (e.g. see fig 5b). The fluorescent intensities linearly increase with time indicating the existence of pseudo first order kinetic reaction. The rate constants of the hydroxylation reactions were estimated from the line's slope of fluorescence intensities against time plots. The calculated rate constant values are illustrated in fig 6.

As shown in fig 6, hydroxylation of coumarin significantly depends on the preparation experimental conditions of $\mathrm{TiO}_{2}$ samples. Overall,

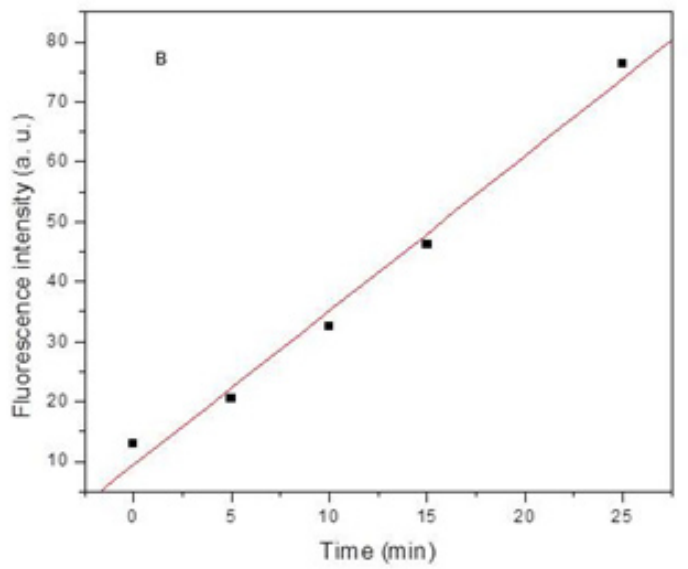

Fig. 5: A: The change in the fluorescence spectrum of coumarin upon illumination in the presence of $\mathrm{TiO}_{2}$ particles $(\mathrm{Ak} 3)$ calcined at $550^{\circ} \mathrm{C}$. B: the corresponding rate constant calculations

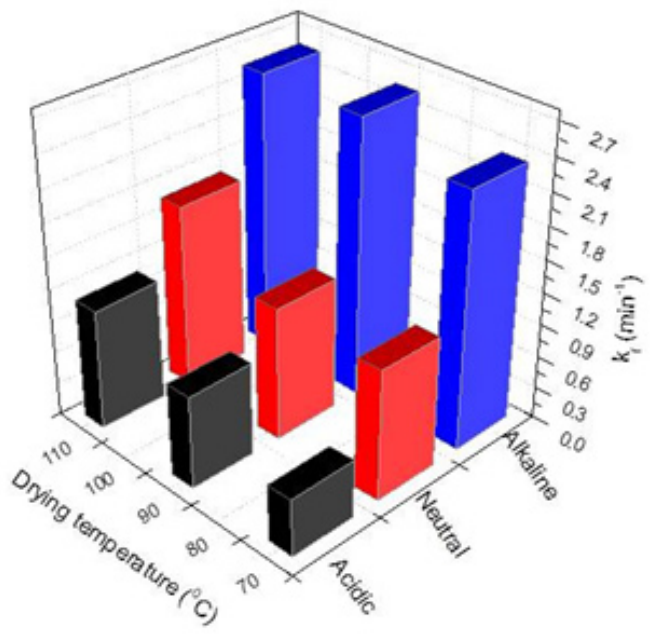

Fig. 6: The Calculated rate constants of the hydroxylation of coumarin using different $\mathrm{TiO}_{2}$ samples the rate of 7-hydroxycoumarin formation apparently increases as the $\mathrm{pH}$ of the preparation media increases. As shown in fig. 6, samples prepared in basic media show comparable rates of formation. The rates of hydroxycoumarin formation; $k_{f}$ are 2.35, 2.57 and $2.58 \mathrm{~min}^{-1}$ for Ak1, Ak2 and Ak3, respectively. This is due to the existence high amount of hydroxyl groups attached to $\mathrm{TiO}_{2}$ surfaces. The high density of surface hydroxyl groups effectively enhances the hydroxylation reaction regardless of the phase or crystallinities of $\mathrm{TiO}_{2}$ samples. In addition, the hydroxylation reaction rates slightly increase as the drying temperatures increase. As given in table 1 , the increase in the drying temperatures in basic media results in the formation of smaller crystal sizes of $\mathrm{TiO}_{2}$. The exposed surfaces of small $\mathrm{TiO}_{2}$ crystals are higher and consequently the reaction rates increase. On the other side, findings were different for samples prepared in both neutral and acidic media. In both neutral and acidic media, the 
hydroxyl radicals' formation is significantly influenced by the drying temperatures. At $\mathrm{pH} 2$, the rates of hydroxycoumarin formation are $0.52,0.86$ and 1.12 $\mathrm{min}^{-1}$ for Ac1, Ac2 and Ac3, respectively. At $\mathrm{pH} 2$, only anatase phase with $50 \%$ degrees of crystallinity was obtained for Ac1 and Ac2 (i. e. samples dried at 70 and $90^{\circ} \mathrm{C}$ ). Samples prepared at these low drying temperatures showed low rates of hydroxylation probably due to their low crystallinities. Meanwhile, the rate of hydroxylation using sample dried at 110 ${ }^{\circ} \mathrm{C}$ (Ac3) is the highest compared to those samples prepared at lower drying temperatures. $\mathrm{TiO}_{2}$ sample dried at $110^{\circ} \mathrm{C}$ shows high degree of crystallinity $(100 \%)$. This consequently results in a relatively high efficiency of hydroxyl radicals' formation. Finally, at $\mathrm{pH} 7$, the order of the rates of hydroxylation were 110 ${ }^{\circ} \mathrm{C}>90^{\circ} \mathrm{C}>70{ }^{\circ} \mathrm{C}$. The rates of hydroxycoumarin formation are 1.20, 1.23 and $1.70 \mathrm{~min}^{-1}$ for Nu1, Nu2 and Nu3, respectively. This finding could be interpreted in terms of the crystal sizes and ratios of rutile to anatase. It has been reported previously that rutile is less photoactive phase than anatase ${ }^{50}$. The prepared $\mathrm{TiO}_{2}$ samples have the same high degrees of crystallinity (100\%) with different sizes and ratios of rutile to anatase phases. Both crystal sizes and rutile phase ratios decrease by increasing the drying temperature. This consequently results in increasing the efficiency of hydroxyl radicals' generation.

\section{CONCLUSION}

In this study, preparation of different structures of $\mathrm{TiO}_{2}$ nanoparticles over narrow ranges of varying parameters was achieved. Titanium-n- butoxide was used to prepare $\mathrm{TiO}_{2}$ nanoparticles via sol gel method. The influence of the experimental preparation conditions: $\mathrm{pH}$, drying and calcination temperatures were studied at ranges 5-9, 70-110 ${ }^{\circ} \mathrm{C}$, and $450-650{ }^{\circ} \mathrm{C}$, respectively. XRD and TEM measurement of the prepared $\mathrm{TiO}_{2}$ indicated the formation of pure anatase, rutile and mixed phases with different ratios. Calcination of samples at $450{ }^{\circ} \mathrm{C}$ results in the formation of only anatase phase with low crystallinity. However, upon calcination at $550^{\circ} \mathrm{C}$, mixed anatase and rutile phases were observed in some samples. Degrees of crystallinity, crystal sizes and phase ratios were significantly influenced by the titanium oxyhydroxid species to water ratios and anions in the reaction media. In addition, increase in the crystallite sizes was observed in samples calcined at $650{ }^{\circ} \mathrm{C}$. The degrees of crystallinity of the prepared samples also increased to $100 \%$. Rutile phase appeared in most samples. Pure rutile phase was obtained at low drying temperature in neutral medium. Finally, fluorescent probe method has been used for initial assessment of the photocatalytic activities of the prepared $\mathrm{TiO}_{2}$ samples. The rate of the hydroxyl radicals' formation on $\mathrm{TiO}_{2}$ surfaces apparently increases as the $\mathrm{pH}$ of the preparation media increases. The hydroxyl radicals' formation is also significantly influenced by the drying temperatures.

\section{ACKNOWLEDGEMENTS}

The science and technology fund (STDF), Egypt, Grant number 4746 supports this work financially.

\section{REFERENCES}

1. Macedo, E. R.; Oliveira, P. S.; de Oliveira, H. P. J. Photochem. Photobiol. A Chem. 2015, 307-308, 108-114.

2. Hazarika, D.; Karak, N.; Appl. Surf. Sci. 2016, 376, 276-285.

3. Wang, W.-Y.; Irawan, A.; Ku, Y. Water Res. 2008, 42, 4725-4732.

4. Mardare, D.; Cornei, N.; Mita, C.; Florea, D.; Stancu, A.; Tiron, V.; Manole, A.; Adomnitei C. Ceram. Int. 2016, 42, 7353-7359.

5. Blank, T.A.; Eksperiandova, L.P.; Belikov K.N. Sens Actuators B Chem. 2016, 228, 416-

\section{2.}

6. Yahui, L.; Fancheng, M.; Fuqiang, F.; Weijing, W.; Jinglong, C.; Tao, Q. Dyes Pigm. 2016, 125, 384-391.

7. Jarosz, M.; Pawlik, A.; Szuwarzyñski, M.; Jaskuª, M.; Sulka, G. D. ýColloids Surf. B. 2016, 143, 447-454.

8. Szymañska, R.; Koºdziej, K.; CElesak, I.; Zimak-Piekarczyk, P.; Orzechowska, A.; Gabruk, M.; ${ }^{-1} \mathrm{~d}^{3} \mathrm{O}$, A.; Habina, I.; Knap, W.; Burda, K.; Kruk, J. Environmental Pollution. 2016, 213, 957-965. 
9. Wang, Y.; Han, W.; Zhao, B.; Chen, L.; Teng, F.; Li, X.; Gao, C.; Zhou, J.; Xie, E. Sol. Energ. Mat. Sol. Cells. 2015, 140, 376-381.

10. Zeynalov, E. B.; Allen, N. S.; Calvet, N. L.; Stratton, J. Dyes Pigm. 2007, 75, 315-327.

11. Nejad, S. M.; Takahashi, H.; Hosseini, H.; Watanabe, A.; Endo, H.; Narihira, K.; Kikuta, T.; Tachibana, K. Ultrason. Sonochem. 2016, 32, 95-101.

12. Kumar, R.; Kushwaha, A. S.; Srivastava, S. K. Optik. 2015, 126, 1324-1330.

13. Pinjari, D.V.; Prasad, K.; Gogate, P. R.; Mhaske, S. T.; Pandit, A. B. Ultrason. Sonochem. 2015, 23, 185-191.

14. Kumar, A.; Mondal, S.; Girish Kumar, S.; Rao, K. S. R. K. Mater. Sci. Semicond. Process. 2015, 40, 77-83.

15. Kumar, N.; Hazarika, S. N.; Limbu, S.; Boruah, R.; Deb, P.; Namsa, N. D.; Das, S. K. Micropor. Mesopor. Mat. 2015, 213, 181-187.

16. Na, S. H.; Shon, H. K.; Kim, J. B.; Park, H. J.; Cho, D. L.; El Saliby, I.; Kim, J.-H. J. Ind. Eng. Chem. 2010, 16, 96-100.

17. Valencia, S.; Vargas, X.; Rios, L.; Restrepo, G.; Marín, J. M. J. Photochem. Photobiol. A Chem. 2013, 251, 175-181.

18. Zangeneh, H.; Zinatizadeh, A. A. L.; Habibi, M.; Akia, M.; Hasnain Isa, M. J. Ind. Eng. Chem.2015, 26, 1-36.

19. Hachisu, T.; Shi, K.; Yokoshima, T.; Sugiyama, A.; Kuroiwa, S.; Osaka, T.; Nakajima, N.; Yoshino, M. Electrochem commun. 2016, 65, 5-8.

20. Du, X.; Wang, X.; You, S.; Wang, Q.; Gong, X. J. Environ. Sci. 2015, 36, 84-92.

21. Dejene, F. B.; Onani, M. O.; Tarus, P. K. Physica B: Condensed Matter. 2016, 480, 213-218.

22. Karaman, M.; Sarýipek, F.; Köysüren, Ö.; Yýldýz, H. B. Appl. Surf. Sci. 2013, 283, 993998.

23. Keswani, R. K.; Ghodke, H.; Sarkar, D.; Khilar, K. C.; Srinivasa, R. S. Colloids Surf. A Physicochem. Eng. Asp. 2010, 69, 75-81.

24. Liu, Z.; Xu, X.; Fang, J.; Zhu, X.; Chu, J.; Li, B. Appl. Surf. Sci. 2012, 258, 3771-3778.

25. Shen, P.-S.; Tseng, C.-M.; Kuo, T.-C.; Shih, C.-K.; Li, M.-H.; Chen, P. Solar Energy. 2015, 120, 345-356.

26. Wang, J.; Pan, Z.; Zhang, Z.; Zhang, X.; Wen, F.; Ma, T.; Jiang, Y.; Wang, L.; Xu, L.; Kang P.
Ultrason. Sonochem. 2006, 13, 493-500.

27. Venkatachalam, N.; Palanichamy, M.; Murugesan V. Mater. Chem. Phys. 2007, 104, 454-459.

28. EL-Mekkawi, D. M.; Galal, H.R.; Abd EL Wahab, R. M.; W. A. A. Mohamed, Int. J. Environ. Sci. Te. 2016, 13, 1077-1088.

29. Hanna, A. A.; Mohamed, W. A. A.; Ibrahim, I. A. Egypt J. Chem. 2014, 57, 315-326.

30. Wang, F.; Feng, L.; Zhang, D.; Tang, Q.; Feng D. J. Alloys Compd. 2014, 611, 125-129.

31. Li, W.; Du, D.; Yan, T.; Kong, D.; You, J.; Li, D.; J. Colloid Interface Sci. 2015, 444, 42-48.

32. Du, J.; Wu, Q.; Zhong, S.; Gu, X.; Liu, J.; Guo, H.; Zhang, W.; Peng, H.; Zou, J. J.Rare Earth. 2015, 33, 148-153.

33. Wang, X.; Sø, L.; Su, R.; Wendt, S.; Hald, P.; Mamakhel, A.; Yang, C.; Huang, Y.; Iversen, B. B.; Besenbacher F. J. Catal. 2014, 310, 100-108.

34. Kanna, M.; Wongnawa, S. Mater. Chem. Phys.2008, 110, 166-175.

35. Prasad, K.; Pinjari, D.V.; Pandit, A.B.; Mhaske, S.T. Ultrason. Sonochem. 2010, 17, 409415.

36. Gandhi, V.G.; Mishra, M.K.; Rao, M.S.; Kumar, A.; Joshi, P.A.; Shah, D.O. J. Ind. Eng. Chem.2011, 17, 331-339.

37. Behnajady, M. A.; Eskandarloo, H.; Modirshahla, N.; Shokri, M. Photochem. Photobiol. 2011, 87, 1002-1008.

38. Karami, A. J. Iran. Chem. Soc. 2010, 7, S154S160.

39. Rajabzadeh, G.; Salehi, S.; Jalalian, A. Synth. React. Inorg. Met.-Org. Chem. 2010, 40, 922-929.

40. Patterson, A. L. Phys. Rev. 1939, 56, 978979.

41. Saif, M.; El-Molla S. A., Aboul-Fotouh, S.M.K.; Hafez, H.; Ibrahim, M.M.; Abdel-Mottaleb, M.S.A, Ismail, L.F.M. Spectrochim. Acta. Mol. Biomol. 2013, 112, 46-51.

42. Burdett, J.K.; Hughbanks, T.; Miller, G.J.; Richardson, J.W.; Smith, J.V. J. Am. Chem. Soc. 1987, 109, 3639 - 3646.

43. Grey, I. E.; Li, C.; MacRae, C. M.; Bursill, L. A. J. solid. State. Chem. 1996, 127, 240-247.

44. Reddy, M. A.; Kishore, M. S.; Pralong, V.; Caignaert, V.; Varadaraju, U.V.; Raveau, B. Electrochem. Commun.2006, 8, 1299-1303. 
45. Hart, J. N.; Bourgeois, L.; Cervini, R.; Cheng, Yi-B. .; Simon, G. P.; Spiccia, L. J. Sol-Gel Sci. Technol.2007, 42, 107-117.

46. Wu, M.; Lin, G.; Chen, D.; Wang, G.; He, D.; Feng, S.; Xu, R. Chem. Mater. 2002, 14, 19741980.

47. Ohno, T.; Sarukawa K.; Matsumura, M. New
J. Chem. 2002, 26, 1167-1170.

48. Ishibashi, K.; Fujishima. A.; Watanabe, T.; Hashimoto, K. Electrochem. Commun. 2000, 2, 207-210.

49. Xiang, Q.; Yu, J.; Wong, P.K. J. Colloid. Interface. Sci. 2011, 357, 163-167.

50. Torimoto, T.; Nakamura, N.; Ikeda, S.; Ohtani, B. Phys. Chem. Chem. Phys. 2002, 4, 59105914. 